Check for updates

Cite this: Chem. Commun., 2018, 54,12726

Received 22nd August 2018, Accepted 15th October 2018

DOI: $10.1039 / \mathrm{c} 8 \mathrm{cc} 06830 \mathrm{e}$

rsc.li/chemcomm

\section{A versatile colloidal Janus platform: surface asymmetry control, functionalization, and applications $\dagger$}

\author{
Chengjiao Wu, ${ }^{a}$ Ziwei Deng, (D) *a Bin Shang, ${ }^{a}$ Olli lkkala (D) ${ }^{b}$ and Bo Peng (D) *bc
}

\begin{abstract}
We show a facile synthetic route towards colloidal $\mathrm{SiO}_{2}$-based Janus particles with tunable asymmetries and functionalities based on the integrated use of silane mixtures, Pickering emulsions, and polydopamine chemistry. We demonstrate the generic nature of the concept and application potential by presenting several functionalities.
\end{abstract}

Janus particles, which generally refer to entities with anisotropic structures and properties, were highlighted and brought to colloidal sciences by Pierre-Gilles de Gennes in his Nobel Prize lecture. ${ }^{1}$ After tepid exploration over about a decade, the research on colloidal Janus particles (roughly $1 \mathrm{~nm}$ to $1 \mu \mathrm{m}$ in size $\mathrm{e}^{2,3}$ ) has recently received considerable attention. ${ }^{4}$ Colloidal Janus particles, a specific case of patchy particles, ${ }^{5-7}$ constitute a unique class of colloids, which integrate broken symmetries in chemical and physical properties allowing for multi-functionalization of materials. ${ }^{4,8-13}$ They can assemble into novel structures ${ }^{4,8,11,13-18}$ leading to a rich portfolio of properties, suitable for a multitude of applications. ${ }^{8,9,12,19-22}$

In general, monodisperse colloidal Janus particles would be desirable with uniform and tunable functional asymmetries, and for mainstream applications a facile scalable synthesis is needed. ${ }^{8,9,11,13,18,23}$ Even if a large variety of synthetic strategies have been developed, e.g., based on self-assembly, seeded emulsion polymerization, and microfluidics, ${ }^{8-12}$ it still remains a challenge to produce large quantities of monodisperse colloidal Janus particles with precise control over the anisotropic properties and functions. Here, we introduce a sequential surface modification by which colloidal Janus particles with adjustable surface

\footnotetext{
${ }^{a}$ Key Laboratory of Applied Surface and Colloid Chemistry, Ministry of Education, Shannxi Key Laboratory for Advanced Energy Devices, Shaanxi Engineering Laboratory for Advanced Energy Technology, School of Materials Science and Engineering, Shaanxi Normal University, Xi'an, 710119, China. E-mail: zwdeng@snnu.edu.cn

${ }^{b}$ Department of Applied Physics, Aalto University, Espoo FI-00076, Finland

${ }^{c}$ Department of Physical and Theoretical Chemistry Laboratory, University of Oxford, South Parks Road, Oxford OX1 3QZ, UK. E-mail: pengbob006@gmail.com $\dagger$ Electronic supplementary information (ESI) available: Experimental section and supporting figures and table. See DOI: 10.1039/c8cc06830e
}

asymmetries and functions can be prepared using a simple process, even suggesting scalable production. These colloidal Janus particles can be multifunctional, e.g., in wastewater treatment, stabilizing oil-in-water emulsions, selectively decomposing water-miscible organic wastes, and collecting immiscible organics from water.

This approach is illustrated for $\mathrm{SiO}_{2}$ particles, as schematically elaborated in Fig. 1a. It consists of three steps and integrates surface wetting tuning, ${ }^{24}$ Pickering emulsification, ${ }^{25}$ and polydopamine (PDA) chemistry. ${ }^{26}$ They are conducted in sequence: (1) first, the highly hydrophilic monodisperse $\mathrm{SiO}_{2}$ colloidal spheres (see Fig. S1, ESI $\dagger$ ) are tunably hydrophobized with silane

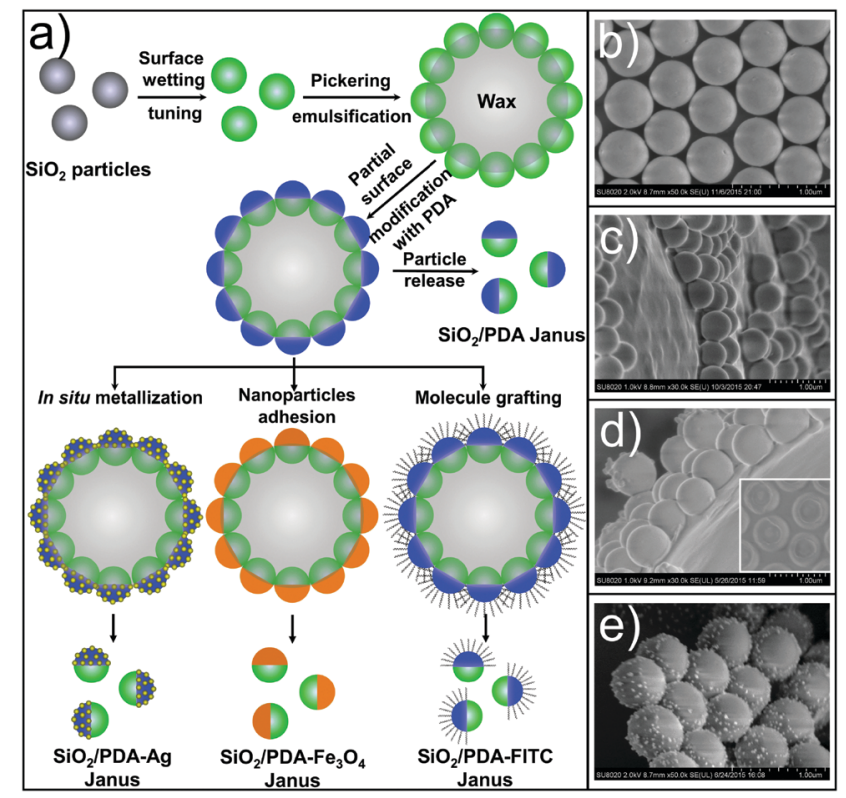

Fig. 1 The synthetic strategy for a versatile colloidal Janus platform: (a) the main steps, i.e., surface wetting tuning, Pickering emulsification, polydopamine (PDA) coating, and functionalization. (b) Scanning electron microscopy (SEM) observations after tuning the surface wetting of the $\mathrm{SiO}_{2}$ particles (mean size of $0.51 \mu \mathrm{m}$, also see Fig. S1a-c (ESI†)). (c) Pickering emulsification, (d) partial surface modification with PDA, and (e) in situ functionalization with Ag nanoparticles. 
mixtures to systematically increase the contact angle to water (takes ca. 2 h); (2) next, such particles are assembled at the waxwater interfaces by Pickering emulsification and immobilized by solidifying the wax (ca. $1 \mathrm{~h}) ;{ }^{27}$ (3) PDA is then introduced onto the partially coated silane-modified $\mathrm{SiO}_{2}$ particles from the aqueous phase to form Janus particles with tunable sizes of PDA patches opposite to the hydrophobic side ( $c a .1 \mathrm{~h}$ ). The PDA patch size depends on the extent of the hydrophobicity brought by the silane mixtures. The chemical reactivity of PDA then serves as a generic chemically reactive platform to introduce a rich variety of functional materials including a catalytic metal (Ag), magnetic metal oxide $\left(\mathrm{Fe}_{3} \mathrm{O}_{4}\right)$ nanoparticles, and fluorescent organic molecules (fluorescein isothiocyanate, FITC). Fig. 1b-e illustrate each step along the approach.

The geometry of the colloidal particles residing at the oilwater interface in Pickering emulsions tightly associates with the surface wetting properties of the particles. ${ }^{28}$ The resident geometry of the particles is quantified in terms of the angle ' $\alpha$ ', while the wetting properties of the particles are reflected by measuring their sessile drop contact angles $(\theta)$ with water, as illustrated in Fig. 2a. As such, the hydrophilic particles $\left(\theta<90^{\circ}\right)$ favour the aqueous phase with $\alpha>90^{\circ}$, while the hydrophobic particles $\left(\theta>90^{\circ}\right)$ tend to lean to the oil phase as $\alpha<90^{\circ} .^{28}$

Achieving stable oil-in-water Pickering emulsions $\left(180^{\circ}>\right.$ $\alpha>90^{\circ}$ ) is subtle. Therein, the surface wetting properties of the particles should be in-depth controllable for $\theta<90^{\circ} .^{28}$ As indicated in Fig. S2 (ESI $\dagger$ ), neither the pristine $\mathrm{SiO}_{2}$ particles (sample A1 in Table 1) nor the hydrophobically dichlorodimethylsilane (DCDMS) treated $\mathrm{SiO}_{2}$ particles (sample A7 in Table 1)

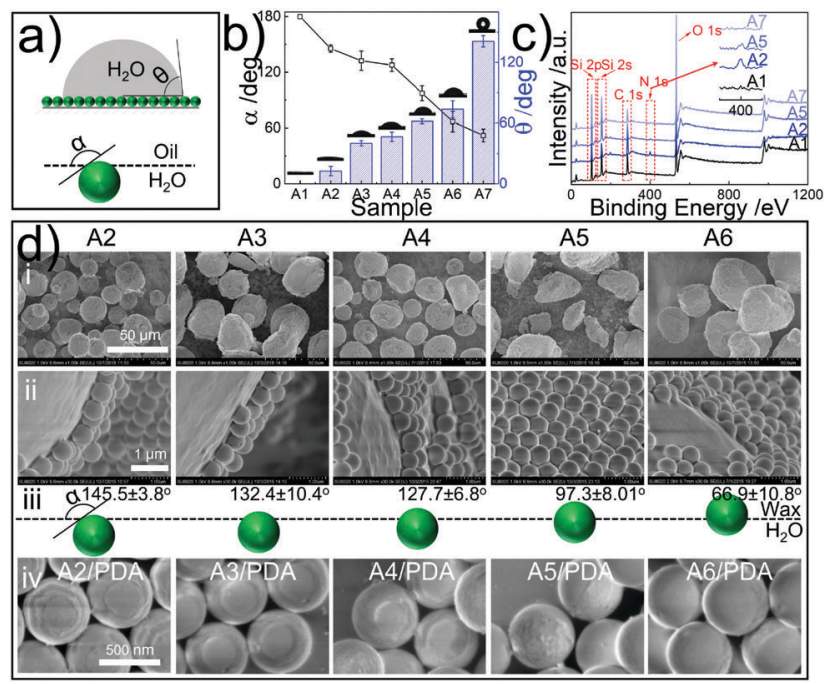

Fig. 2 Topological tuning of the Janus $\mathrm{SiO}_{2}$ colloids. (a) Schematics for $\theta$ (water sessile drop contact angle) and $\alpha$ (used for quantifying the resident geometries of the particles at the oil/water interfaces). (b) $\alpha$ and $\theta$ for the samples presented in Table 1; (c) X-ray photoelectron spectroscopy (XPS) results of samples $A 1, A 2, A 5$ and $A 7$, see also Fig. S5 (ESI $\dagger$ ). The insets highlight the characteristic patterns of N1s in each sample; (d) SEM observations of wax droplets stabilized by silane-modified $\mathrm{SiO}_{2}$ particles (i, A2-A6), magnified local details (ii), and $\mathrm{SiO}_{2} / \mathrm{PDA}$ Janus particles (iv, A2A6/PDA), and schematic illustration of particle resident geometries at the wax-water interfaces where $\alpha$ is specifically defined (iii).
Table 1 The concentration ratios $\left(C_{\text {APTES }} / C_{\text {DCDMS }}\right)$ of (3-aminopropyl)triethoxysilane (APTES) and dichlorodimethylsilane (DCDMS), in relation to the detailed recipes in Table S1 (ESI), and the PDA patch areas as a fraction of the total particle surface area $\left(S_{\mathrm{PDA}} / S_{\text {total }}\right)$

\begin{tabular}{lll}
\hline Sample & $C_{\text {APTES }} / C_{\text {DCDMS }}[\mathrm{mol}: \mathrm{mol}]$ & $S_{\text {PDA }} / S_{\text {total }}[\%]$ \\
\hline A1 & - & 1 \\
A2 & $1: 0$ & $91.0 \pm 2.0$ \\
A3 & $1: 0.0636$ & $83.7 \pm 6.1$ \\
A4 & $1: 0.191$ & $80.6 \pm 4.5$ \\
A5 & $1: 0.381$ & $56.4 \pm 7.0$ \\
A6 & $1: 0.636$ & $30.4 \pm 9.0$ \\
A7 & $0: 1$ & 0
\end{tabular}

stabilize oil-in-water Pickering emulsions. The pristine $\mathrm{SiO}_{2}$ particles are superhydrophilic (Fig. S2a, ESI $\dagger$ ) with a $\theta \approx 0^{\circ}$ (Fig. 2b), favouring fully staying in the aqueous phase $\left(\alpha=180^{\circ}\right)$, while the DCDMS-treated particles are highly hydrophobic $\left(\theta=140.9 \pm 5.6^{\circ}\right.$, see Fig. $\left.2 \mathrm{~b}\right)$ and tend to form water-in-oil Pickering emulsions (Fig. S2b, ESI $\dagger$ ). Therefore, silane mixtures comprising both DCDMS and (3-aminopropyl)triethoxysilane (APTES) were assumed to allow the formation of surfaces with tunable wetting properties. Note that APTES is expected to co-react with DCDMS and to introduce hydrophilic $-\mathrm{NH}_{2}$ and $-\mathrm{OH}$ groups (see Fig. S3 and S4a, ESI $\dagger$ ).

The concentration ratios of APTES and DCDMS $\left(C_{\mathrm{APTES}} / C_{\mathrm{DCDMS}}\right)$ are systematically varied and detailed in Table 1 (also see Table S1, $\mathrm{ESI} \dagger$ ). As expected, APTES assists the formation of hydrophilic surfaces (see sample A2 in Fig. 2b), while DCDMS brings $-\mathrm{CH}_{3}$ (see Fig. S4a, ESI $\dagger$ ), enhancing the hydrophobicity of the surfaces (Fig. 2b). Therefore, the integrated use of both silanes gives rise to surfaces with intermediate wetting properties. X-ray photoelectron spectroscopy (XPS) reveals the co-grafting of APTES and DCDMS, and verifies the role of DCDMS as the hydrophobicity enhancer, as demonstrated in Fig. 2c and Fig. S5 (ESI $\dagger$ ). Further results from Fourier-transform infrared spectroscopy (Fig. S4a, $\mathrm{ESI} \dagger$ ), zeta potential analysis (Fig. S4b, ESI $\dagger$ ), size comparison from SEM observation (Fig. S4c, ESI $\dagger$ ), and dispersibility measurements (Fig. S4d, ESI $\dagger$ ) corroborate those revealed by XPS. More importantly, increasing the $C_{\mathrm{APTES}} / C_{\mathrm{DCDMS}}$ ratio from $1: 0$ to $1: 0.636$ leads to an increase of $\theta$ from 12.6 to $73.7^{\circ}$, and a decline of $\alpha$ from 145.5 to $67^{\circ}$ as indicated in Fig. $2 \mathrm{~b}$ and d-iii. Correspondingly, the fractional area of the PDA patch $v s$. the particle total surface decreases from $91.0 \%$ to $30.4 \%$, as displayed in Table 1 . Clearly, $\mathrm{SiO}_{2}$ particles are capable of stabilizing oil-in-water Pickering emulsions upon treatment with APTES and DCDMS in the $C_{\text {APTES }} / C_{\text {DCDMS }}$ range from 1:0 to $c a$. 1:0.636 (Fig. $2 \mathrm{~d}$-i and Fig. S6, ESI $\dagger$ ), whereupon their resident geometries at the interfaces become $C_{\mathrm{APTES}} / C_{\mathrm{DCDMS}}$ dependent (Fig. 2d-ii). The particles tend to lean into the wax phase as an increased composition of $C_{\mathrm{APTES}} / C_{\mathrm{DCDMS}}$ is applied as shown in Fig. 2d-ii. Upon solidifying the wax phase via lowering the temperature, the particles are fixed at the interfaces, allowing subsequent topochemical PDA modification of the patches on the particle surface that are exclusively immersed in the water phase (Fig. 2d-i, ii and Fig. S6, ESI $\dagger$ ). After the removal of the wax with chloroform, a series of $\mathrm{SiO}_{2} / \mathrm{PDA}$ Janus particles are formed as shown in Fig. 2d-iv (also see the relevant characterizations in Fig. S7, ESI $\dagger$ ). 
PDA is abundant in catechol and amino groups, allowing for a rich variety of secondary functionalizations..$^{26,29,30}$ Here, we demonstrate the generality of the approach based on three types of surface functionalization based on PDA patches, i.e., (1) in situ metallization, ${ }^{31}$ (2) nanoparticle adhesion, and (3) molecule grafting. First, the PDA patches facilitate in situ reduction of $\mathrm{Ag}^{+}$ ions to $\mathrm{Ag}$ nanoparticles (about $10 \mathrm{~nm}$ in diameter, see Fig. $3 \mathrm{~b}$ and Fig. S8, ESI $\dagger$ ), resulting in $\mathrm{SiO}_{2}$ /PDA-Ag Janus particles with variable asymmetrical geometries as shown in Fig. 3a. The formation of $\mathrm{SiO}_{2} / \mathrm{PDA}-\mathrm{Ag}$ Janus particles is verified with transmission electron microscopy (TEM, Fig. 3b-i and ii, and Fig. S8a-c, ESI $\dagger$ ), energy-dispersive X-ray spectroscopy (EDX, Fig. S8d, ESI $\dagger$ ), elemental mapping (Fig. 3b-iii), XPS (Fig. 3b-iv), and X-ray diffraction (XRD, Fig. 3b-v).

Second, $\mathrm{Fe}_{3} \mathrm{O}_{4}$ nanoparticles stabilized with trisodium citrate can be adhered exclusively onto PDA patches by simply mixing $\mathrm{Fe}_{3} \mathrm{O}_{4}$ nanoparticles with the $\mathrm{SiO}_{2} / \mathrm{PDA}$ Janus particles in water (for details see Section S1.2.6 in the ESI $\dagger$ ), yielding $\mathrm{SiO}_{2} / \mathrm{PDA}-\mathrm{Fe}_{3} \mathrm{O}_{4}$ Janus particles as evidenced by TEM (Fig. 3c-i, ii and Fig. S9a, b, ESI $\dagger$ ), elemental mapping (Fig. S9c, ESI $\dagger$ ), EDX (Fig. S9d, ESI $\dagger$ ), XRD (Fig. 3c-iii), and XPS (Fig. S10, ESI $\dagger$ ). $\mathrm{SiO}_{2} / \mathrm{PDA}-\mathrm{Fe}_{3} \mathrm{O}_{4}$ Janus particles are superparamagnetic at room

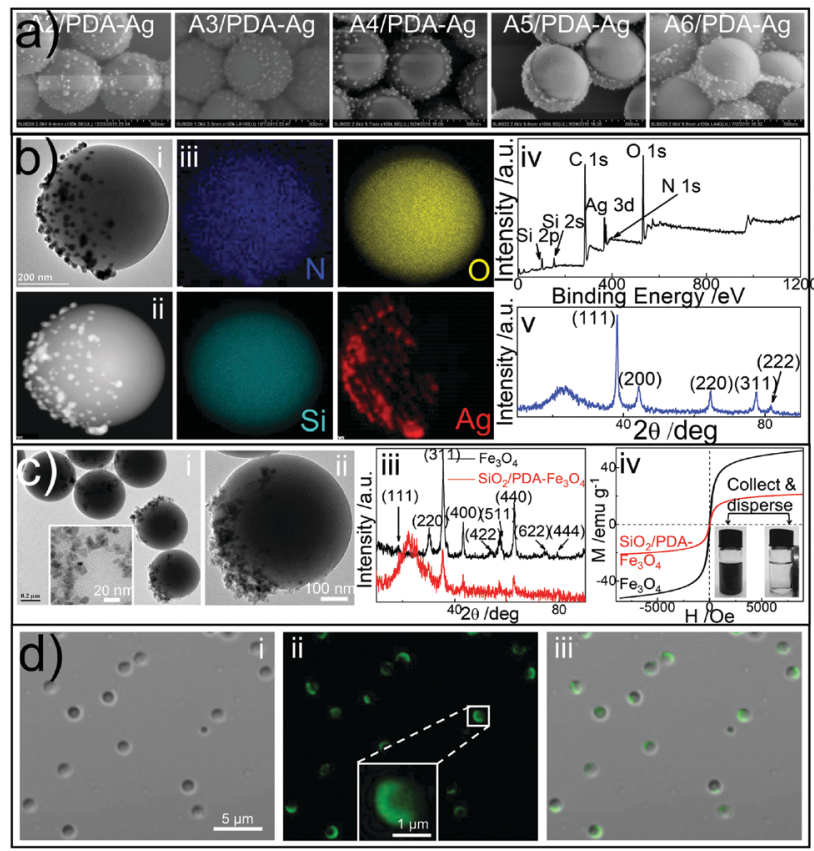

Fig. 3 Surface functionalization of $\mathrm{SiO}_{2} / \mathrm{PDA}$ Janus particles. (a) Morphology control of $\mathrm{SiO}_{2} / \mathrm{PDA}-\mathrm{Ag}$ particles (A2-A6/PDA-Ag were prepared based on the samples A2-A6/PDA shown in Fig. 2d). (b) TEM (i), dark field TEM (ii), elemental mapping (iii), XPS (iv), and XRD (v) characterization of $\mathrm{SiO}_{2} / \mathrm{PDA}-\mathrm{Ag}$ Janus particles prepared through in situ metallization. (c) TEM (i), dark field TEM (ii), XRD (iii), and magnetic (iv) measurements of $\mathrm{SiO}_{2} / \mathrm{PDA}-\mathrm{Fe}_{3} \mathrm{O}_{4}$ Janus particles synthesized via nanoparticle adhesion. The insets in (i) and (iv) demonstrate the $\mathrm{Fe}_{3} \mathrm{O}_{4}$ nanoparticles used and the magnetic collection and dispersion of $\mathrm{SiO}_{2} / \mathrm{PDA}-\mathrm{Fe}_{3} \mathrm{O}_{4}$ Janus particles, respectively. (d) Optical (i), confocal laser scanning microscopy (CLSM, ii), and their integrated micrographs of $\mathrm{SiO}_{2} / \mathrm{PDA}$-FITC Janus particles produced by grafting fluorescein isothiocyanate (FITC) molecules. Scale bars are shared in the same sections unless otherwise denoted. temperature (Fig. 3c-iv). They are promising candidates for magnetically recyclable applications. ${ }^{32,33}$

Third, we further demonstrate the coupling of dye molecules, e.g., fluorescein isothiocyanate (FITC) with PDA shells (see Section S1.2.7 in the ESI $\dagger$ ). As displayed in Fig. 3d, the surfaces of $\mathrm{SiO}_{2} / \mathrm{PDA}$ particles are partially fluorescent after grafting, indicating the formation of $\mathrm{SiO}_{2} / \mathrm{PDA}-\mathrm{FITC}$ Janus particles (also see Fig. S11, ESI $\dagger$ ). Note that the present functionalization is a general strategy regardless of the sizes of the colloidal particles (Fig. S12, ESI $\dagger$ ).

Finally, we demonstrate how Janus particles can be feasible to selectively capture and then decolorize oils from wastewater, facilitating the wastewater remedy by avoiding the combined employment of a number of conventional colloidal materials that only play a single role during the remedy. ${ }^{34}$ To simulate the wastewater, water-soluble (4-nitrophenol) and -immiscible oil (red-stained toluene) organics are mixed with water (see Fig. 4a-i). After the employment of $\mathrm{SiO}_{2} / \mathrm{PDA}-\mathrm{Ag}$ Janus particles, the oil droplets are surrounded with Janus particles in the formation

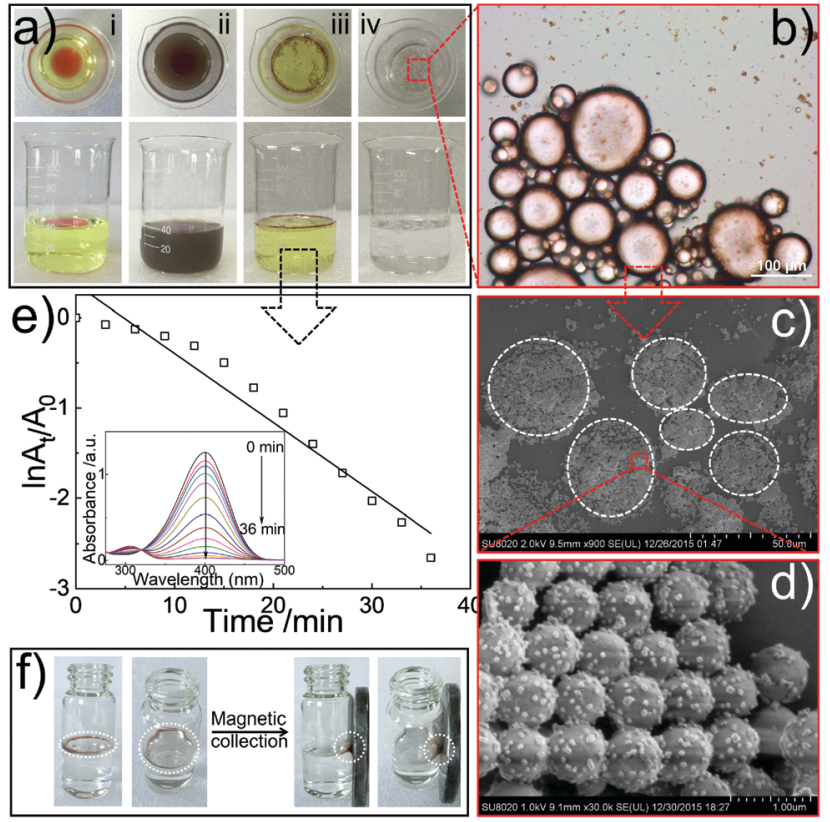

Fig. 4 Demonstration of Janus particles in wastewater remedy. (a) Photographs of wastewater remedy with $\mathrm{SiO}_{2} / \mathrm{PDA}-\mathrm{Ag}$ Janus particles. (i) The simulated wastewater consisting of red-stained toluene (oil) and watersoluble 4-nitrophenol, (ii) the addition of the Janus particles, (iii) toluene droplets protected by Janus particles, forming Pickering emulsions, and (iv) the selective decomposition of 4-nitrophenol with Janus particles. (b) Optical micrograph of Pickering emulsions stabilized by Janus particles after catalysis, where toluene droplets remain reddish while the colour of the aqueous phase fades. SEM observations ( $c$ and $d$ ) of Janus particles staying at interfaces. Note that Pickering emulsion droplets collapse after drying, their shapes are guided by the white dashed circles in (c), and the surfaces of the Janus particles coated with PDA-Ag face to the aqueous phase (d). (e) The normalized $A_{t} / A_{0} U V$-vis absorption maxima of 4-nitrophenol dissolved in the aqueous phase during the catalysis, where $A_{0}$ and $A_{t}$ are the initial and real time intensities, respectively. The inset shows the pristine data. The line only guides the eyes. (f) Magnetic collection of oil droplets from the aqueous phase based on $\mathrm{SiO}_{2} / \mathrm{PDA}$ $\mathrm{Fe}_{3} \mathrm{O}_{4}$ Janus particles. 
of Pickering emulsion (Fig. 4a-iii, b and c). The surface of the particle decorated with PDA-Ag faces to the aqueous phase (Fig. 4c and d), allowing the catalysis to occur exclusively within the aqueous phase. Clearly, 4-nitrophenol is decomposed gradually as the absorbance of 4-nitrophenol decreases (Fig. 4e) and the yellow colour in the aqueous phase fades (Fig. 4a-iv), while the red colour in oil is retained (Fig. 4b). Furthermore, the employment of $\mathrm{Fe}_{3} \mathrm{O}_{4}$ nanoparticles facilitates magnetic enrichment and collection of oils (Fig. 4f), which can be applied for oil recovery from water.

In conclusion, we demonstrate a straightforward and versatile synthetic approach for colloidal Janus particles that allows tuning of the surface asymmetries and functions relying on the usage of silane mixtures combined with a Pickering emulsion technique, as well as PDA chemistry. The facile chemistry paves ways towards bulk synthesis. We also demonstrate that these Janus particles are promising candidates for purifying wastewater as the Pickering emulsifiers, the catalysts for the selective decomposition of watersoluble organics, and the magnetic vectors for recycling oils. This facile synthesis, for the first time, integrates the three techniques as a complete platform for preparing colloidal Janus particles with tunable topologies and sticky reactive patches that allow for generic functionalization relying on the requirements of the applications. This colloidal Janus platform may also be adapted for other fields, for instance, drug delivery and active matter.

The financial support from the National Natural Science Foundation of China (No. 51473089), the Natural Science Basic Research Plan in Shaanxi Province of China (No. 2018JM5093), the Science and Technology Program of Shaanxi Province (No. 2015KJXX-20), the Program for Science \& Technology Innovation Team of Shaanxi Province (No. 2018TD-030), and the Fundamental Research Funds for the Central Universities (No. GK201702009) for this research is appreciated. ERC Adv. Grant (DRIVEN 2017-2022) in the finalization stage is acknowledged.

\section{Conflicts of interest}

There are no conflicts to declare.

\section{Notes and references}

1 P.-G. De Gennes, Angew. Chem., Int. Ed., 1992, 31, 842-845.
2 Y. Xia, B. Gates, Y. Yin and Y. Lu, Adv. Mater., 2000, 12, 693-713.

3 D. H. Everett, Basic Principles of Colloid Science, The Royal Society of Chemisty, London, 1988.

4 J. Zhang, B. A. Grzybowski and S. Granick, Langmuir, 2017, 33, 6964-6977.

5 A. B. Pawar and I. Kretzschmar, Macromol. Rapid Commun., 2010, 31, 150-168.

6 A. H. Gröschel, A. Walther, T. I. Löbling, F. H. Schacher, H. Schmalz and A. H. E. Müller, Nature, 2013, 503, 247-251.

7 Y. Wang, Y. Wang, D. R. Breed, V. N. Manoharan, L. Feng, A. D. Hollingsworth, M. Weck and D. J. Pine, Nature, 2012, 491, 51-55.

8 A. Walther and A. H. E. Müller, Chem. Rev., 2013, 113, 5194-5261.

9 J. Hu, S. Zhou, Y. Sun, X. Fang and L. Wu, Chem. Soc. Rev., 2012, 41, 4356-4378.

10 A. Walther and A. H. E. Müller, Soft Matter, 2008, 4, 663-668.

11 S. Jiang, Q. Chen, M. Tripathy, E. Luijten, K. S. Schweizer and S. Granick, Adv. Mater., 2010, 22, 1060-1071.

12 A. Perro, S. Reculusa, S. Ravaine, E. Bourgeat-Lami and E. Duguet, J. Mater. Chem., 2005, 15, 3745-3760.

13 J. Zhang, E. Luijten and S. Granick, Annu. Rev. Phys. Chem., 2015, 66, 581-600.

14 Q. Chen, J. K. Whitmer, S. Jiang, S. C. Bae, E. Luijten and S. Granick, Science, 2011, 331, 199-202.

15 J. Yan, M. Bloom, S. C. Bae, E. Luijten and S. Granick, Nature, 2012, 491, 578-581.

16 S. Jiang, J. Yan, J. K. Whitmer, S. M. Anthony, E. Luijten and S. Granick, Phys. Rev. Lett., 2014, 112, 218301.

17 J. Yan, S. C. Bae and S. Granick, Adv. Mater., 2015, 27, 874-879.

18 J. Palacci, S. Sacanna, A. P. Steinberg, D. J. Pine and P. M. Chaikin, Science, 2013, 339, 936-940.

19 Y. Sun, M. Chen, Z. Wang and L. Wu, Chem. Commun., 2014, 50, 5767-5770.

20 C. Xu, B. Wang and S. Sun, J. Am. Chem. Soc., 2009, 131, 4216-4217.

21 S. Hu and X. Gao, J. Am. Chem. Soc., 2010, 132, 7234-7237.

22 B. Liu, W. Wei, X. Qu and Z. Yang, Angew. Chem., Int. Ed., 2008, 47, 3973-3975.

23 A. Walther, M. Hoffmann and A. H. E. Müller, Angew. Chem., Int. Ed., 2008, 120, 723-726.

24 C. J. Brinker and G. W. Scherer, Sol-Gel Science: The Physics and Chemistry of Sol-Gel Processing, Academic Press, Inc., 1990.

25 S. U. Pickering, J. Chem. Soc., 1907, 91, 2001-2021.

26 H. Lee, S. M. Dellatore, W. M. Miller and P. B. Messersmith, Science, 2007, 318, 426-430.

27 L. Hong, S. Jiang and S. Granick, Langmuir, 2006, 22, 9495-9499.

28 R. Aveyard, B. P. Binks and J. H. Clint, Adv. Colloid Interface Sci., 2003, 100-102, 503-546.

29 Z. Deng, B. Shang and B. Peng, Chem. Rec., 2018, 18, 410-432.

30 Y. Liu, K. Ai and L. Lu, Chem. Rev., 2014, 114, 5057-5115.

31 Y. Sheng, G. Sun and T. Ngai, Langmuir, 2016, 32, 3122-3129.

32 A. H. Lu, E. L. Salabas and F. Schüth, Angew. Chem., Int. Ed., 2007, 46, 1222-1244.

33 B. Peng, X. Zhang, D. G. A. L. Aarts and R. P. A. Dullens, Nat. Nanotechnol., 2018, 13, 478-482.

34 X. Qu, P. J. J. Alvarez and Q. Li, Water Res., 2013, 47, 3931-3946. 